were in support of this. The original application of restriction enzyme analysis of mtDNA, namely to detect differences in fragment patterns between strains and mutants of yeast, was extended by Barnardi (Paris) in an elegant demonstration of recombination of mitochondrial genomes in yeast crosses: physical evidence of recombinational events was clearly seen in vegetative segregants with new fragment patterns compared with those of the parental strains. On purely statistical grounds, Bernardi drew the interestine conclusion that cleavage sites of restriction endonucleases may be GC-rich segments of the genome which are not themselves coding regions. Four papers were concerned with the complexity of kinetoplast DNA of trypanosomes. Endonuclease treatment and electron microscope studies indicated that the strange network of KDNA may be made up of aggregates and concentrates of several thousand mini-circles which individually have a contour length of $0.3 \mu \mathrm{m}$. However, there may be more than one class of mini-circle as indicated by sequence heterogeneity, more than one sedimentation peak and little renaturation of mini-circle preparations.

The mtDNA of Paramecium aurelia is circular but the circles may vary in size (Cummings, Colorado). Borst (Amsterdam) could provide no evidence that the mtDNA of Tetrahymena is other than linear and showed that replication intermediates are also linear, so this organism remains the odd one out in this respect. Systems for transference of mitochondrial type from one cell to another in Paramecium (Tait, Edinburgh) and in mammalian tissue culture (Eisenstadt, New Haven) were described as well as a system for the expression of mouse mtDNA in prokaryotic and eukaryotic cells (Clayton, Stanford).

\section{A million memories?}

\section{from Andrew Holmes-Siedle}

SOLID-STATE physicists have been refining their views on how far the miniaturisation of semiconductor circuitry can go (Wallmark, Institute Phys. Conf. Ser. No. 25, 133; 1975 and Keyes, Proc. Inst. Elect. Engng, 63, $740 ; 1975$ ) and it is thus worth seeing how near the integrated-circuit industry is coming to the physical limits which have been defined. Such is the urgency of the competitive rush to cram circuits on to silicon chips in larger and larger numbers and to operate them at high rates that it would seem that, if the rate of size reduction maintained its pace, designers would soon face funda- mental barriers such as the speed of light, the onset of avalanche breakdown in semi-conductors and the randomness of impurity distribution. The field of semiconductor memories is at the centre of this competition.

The electronics journals are pulsating with advice and controversy over which of the dozens of designs of silicon memory chip are the best to use. Chips measuring $0.3 \times 3 \times 3 \mathrm{~mm}$ and carrying 4,096 bits of information can now be made cheaply (say for £5) and there is speculation that next year the semiconductor industry may be able to mass produce a chip containing 16,384 bits with little increase in chip size. Both devices are in demand for installation into new minicomputers and, in addition, all the logical operations will soon be performed on a single "central processor" chip (the "microcomputer"). Also, advanced technology laboratories are already hard at work on the

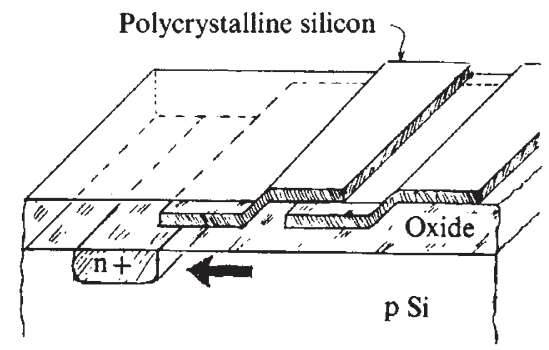

Fig. 1 Two level electrodes.

65,536-bit $\left(2^{16}\right.$-bit $)$ memory chip, which might be produced by 1979 .

I wish to show that in this "memory chip" field, we may get all we need in the way of storing information in a small space without meeting limitations due to physical laws. I submit that we probably store about a million $\left(2^{20}\right)$ bits on a single chip of silicon only just over half an inch on a side (say $15 \times 15 \mathrm{~mm}$ ) without great efforts and that we will then probably stop trying to go further because this capacity is adequate for most purposes where miniature size and low weight are important, and the cost of even greater density may be prohibitive.

As I have discussed before (Nature, 257, 739; 1975), several physical constraints come in when the width of an

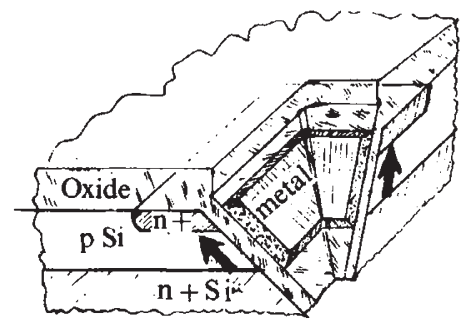

Fig. 2 Use of depth in Si.

individual line in the circuit pattern becomes less than about $2 \mu \mathrm{m}$ (for example if a particular diffused junction region must have dimensions less than $2 \times 2 \mu \mathrm{m})$. Several tricks in metaloxide-semiconductor (MOS) device technology, however, have been devised which make it possible to pack in more functions per square micrometre without reducing the individual line widths. First, the use of multilayer conductor geometry enables two electrodes to overlap (Fig. 1); second, the surface of the silicon is now being etched into grooves (Fig. 2), which leads to the first example of a vertical MOS device, and third, the number of electrodes needed to perform a function is being reduced by the invention of new device principles. For example, using the memory principle shown in Fig. 3, the charge-coupled random access memory (CC-RAM) it seems possible for information to be stored in a cell of $100 \mu \mathrm{m}^{2}(10 \times 10 \mu \mathrm{m})$ or smaller. The cell structure is so simple that the individual line widths used need not be less than $3 \mu \mathrm{m}$. As $1 \mathrm{~cm}^{2}$ is $10^{8} \mu \mathrm{m}^{2}$, it is easy to get a million bits on to an area smaller than a thumbnail. In fact, allowing extra 'real estate' for leads, amplifiers and wiring contacts, the

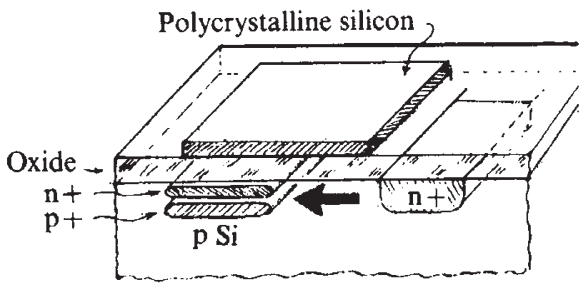

Fig. 3 Use of new electrodeless effects.

million-bit chip would probably be $15 \times 15 \mathrm{~mm}$ or $0.6 \times 0.6 \mathrm{inch}$. If we consider about a thousand of these chips mounted in individual ceramic packages and then on circuit boards, and stacked in the corner of a room, we could easily imagine a capacity of a few billion bits of memory in a small cabinet. Would we want to go further in capacity in this form? Probably not; the Encyclopaedia Britannica only contains 40 million words and any larger storage requirement, say the keeping of archives or the storing of video information (a billion bits is only a few video frames), can quite often be handled by the use of disks or tapes.

This is only to say then that, in the semiconductor field, the basic device phenomena are available to take us to the small "mass memory" density needed in small, portable computers. There will probably be at least half a decade of technological effort before the million-bit density is achieved at reasonable cost. The technology which will challenge magnetic tapes will be of an entirely different character and will probably involve light holography. 